\title{
Towards Modeling Human Arm Movement in a CVE
}

\author{
Fred Stakem \\ Georgia Institute of Technology \\ 210 Technology Circle \\ Savannah, GA 31407 \\ fstakem@gatech.edu
}

\author{
Ghassan AIRegib \\ Georgia Institute of Technology \\ 210 Technology Circle \\ Savannah, GA 31407 \\ gregib@ece.gatech.edu
}

\author{
Biing-Hwang (Fred) Juang \\ Georgia Institute of Technology \\ 777 Atlantic Drive \\ Atlanta, GA 30332 \\ juang@ece.gatech.edu
}

\begin{abstract}
Although Collaborative Virtual Environments share many characteristics in common with first person 3D games, CVEs strive for a deeper level of immersion by networking together individuals with more natural input devices. More complex input devices such as data gloves and positional trackers have additional processing delay and when compounded with network delay can cause CVE applications to lag and become jerky. The purpose of this experiment was to measure and model typical movements from users utilizing data gloves and positional trackers so that algorithms can be designed to help combat the inherent delay and jitter in a CVE. Most movements studied were shown to have a rough Gaussian shaped speed profile, but intricate manipulative movements exhibited greater peaks and valleys than typical reaching movement.
\end{abstract}

\section{Categories and Subject Descriptors}

I.6.5 [Simulation Modeling]: Model Development - modeling methodologies.

\section{General Terms}

Measurement, Documentation, Design, Experimentation, Human Factors, Theory.

\section{Keywords}

Collaborative Virtual Environment, Virtual Worlds, Networking

\section{INTRODUCTION}

The proceedings are the records of the conference. ACM hopes to In a $3 \mathrm{D}$ virtual world the manner in which the user interacts with the $3 \mathrm{D}$ space is governed by the design of the hardware and software. Interaction in a CVE is rich by design focusing on a small immersive virtual environment and allowing more natural forms of input [4] [7]. Typically in a CVE the user will interact with the environment using specialized VR equipment such as

Permission to make digital or hard copies of all or part of this work for personal or classroom use is granted without fee provided that copies are not made or distributed for profit or commercial advantage and that copies bear this notice and the full citation on the first page. To copy otherwise, to republish, to post on servers or to redistribute to lists, requires prior specific permission and/or a fee. IMMERSCOM 2007, October 10-12, Verona, Italy

Copyright $\odot 2007$ 978-963-9799-06-6

DOI 10.4108/ICST.IMMERSCOM2007.2082

data gloves and positional trackers. The use of data gloves and positional trackers allows for a greater degree of input freedom than a game pad or computer mouse, and they do not require the user to translate a human action into an artificial input movement. It is the job of the CVE software to digest the information from the hardware sensors and use it control to the user's avatar in the virtual world.

With new and more complex input devices comes the need to better understand and process input. Delay and delay variation between the user's actual movements and those exhibited in the virtual environment can cause a noticeable lag or jerkiness to the user's avatar in the 3D world [5] [10] [11] [13] [16]. Any lag or irregularity of movement is quickly noticed by the user and reduces realism and immersion in the environment [9]. Currently there are two general sources of delay and jitter in a CVE. The first source occurs in the VR hardware itself. Collecting, processing, and finally transmitting information from the external sensors to the CVE software can produce a noticeable delay. Since the delay is part of the hardware a designer of a CVE would have no means to reduce it and must therefore deal with its effects.

Delay and jitter in CVEs are also caused by the networking of CVE systems together. The purpose of a CVE is to allow remote users to interact in an immersive fashion as if they were located in the same room or work place. To simulate this form of interaction the CVEs must be networked together from often distant locations using Internet protocols. As with hardware delay and jitter, the software designer of a CVE has no means to reduce the network delay and jitter and must design algorithms to compensate for it. One such technique used in networked gaming applications is called dead reckoning [1] [12]. It is a predictive technique for estimating the location of an object in the future given its past position, velocity, and perhaps acceleration. Although dead reckoning works well for gaming applications, its main purpose it to reduce network traffic and has not been proven to work for more complex human movement patterns. Other predictive techniques have been studied for use in virtual reality, but no consensus has been made as the most appropriate method [8].

Before intelligent algorithms can be designed to reduce delay and jitter, a thorough understanding of the input signal's characteristics needs to be made. By studying and classifying input patterns a more accurate model of user input can be deduced and experimented with. Since data gloves and positional tracker technology has matured they have been the primary device used for input into CVEs. To classify all different types of human movement possible with these devices would be a monumental task, but certain common movements and patterns will show up in

Many different applications. Specifically when users collaborate they often reach for items and manipulate the items once they are grasped. The process of reaching for an object is a core movement used in many tasks and has been extensively studied in neurology and robotics research. Reaching for specific positions has been 
shown to produce Gaussian speed profiles in the user's hands [6]. The Gaussian speed profile exhibits a smooth movement readily discriminable by humans. Sharp and jerky movements characteristic of digital inputs such as game pads used in many FPS are easily recognized by users as not being natural. It has yet to be shown if the techniques and analysis used in the growing field of network gaming will translate smoothly to virtual environments which have more human movement patterns [2] [15] [17].

\section{PRODECURE}

Designing a CVE that takes into account all types of movement is impossibility, but a well designed CVE will be flexible enough to allow many different types of interaction. The CVE designed and built for the experiment was a compromise between the flexibility and the complexity of the prototype. By keeping the virtual environment to a single room, the focus of the CVE was on close interaction which is vital for collaboration. In the main virtual environment workspace many 3D background objects were designed for aesthetic purposes, but the users limited their interaction to a table top in the center of the room.

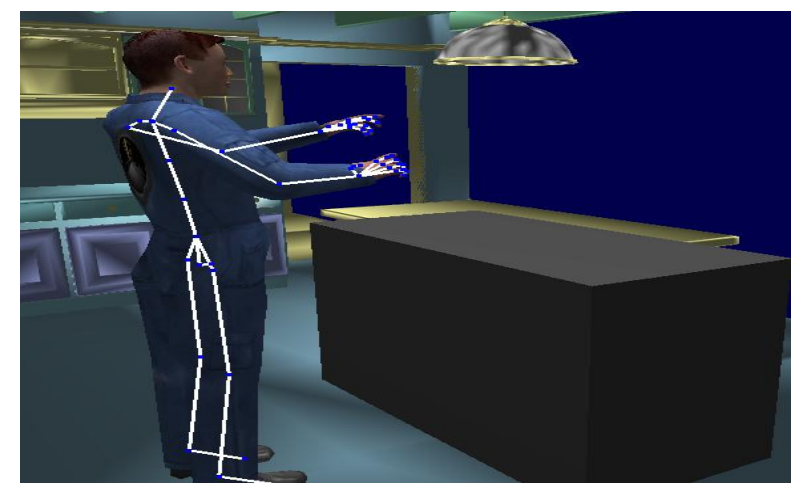

Fig 1: View of Virtual Environment

To interact in the CVE the users donned 5DT data gloves and Nest of Birds magnetic trackers. The 5DT data gloves allow realistic finger articulation while the Nest of Birds trackers measured the position of the user's hands, arms, and torso. A large 21' flat panel monitor was used for viewing the virtual environment because it allowed for users to easy reorient themselves when switching between tasks in the real world and tasks in the virtual world. By using a monitor instead of a HMD the additional delay caused a HMD could also be avoided.

Instead of having the users perform a very specific task, such as hammering a nail, the CVE was designed to focus on general forms of movement. In keeping with this concept, application specific tools were not introduced into the experiments, but the main objects used for interaction were virtual blocks. Using blocks instead of specific tools allowed the users to arrange the virtual objects in many different ways and to build many different structures which is not possible with a tool designed for one task. Although this limited the types of movements and tasks a user can perform, a great deal of common movements can still be done in this environment. Different size and shape blocks were used in some preliminary trials to give the user a wider array of types of objects to handle.
Before any measurements were made, the CVE was tested with various users performing tasks in open experimentation. Users were first asked to perform a task in the real world with blocks, and then they were asked to replicate the task in the virtual environment. When asked to asked to reach for objects users had no problem in the real world, and once oriented no problem in the virtual environment. Users were also asked to manipulate the blocks to build various structures. For example one task was to arrange the blocks into a square, and another task was to stack the blocks on top of each other into a tower. In this case the movement in the real world was relatively effortless, while the task in the virtual environment was difficult and took longer for the users to complete. The accuracy needed for intricate tasks with a great deal of small manipulations was more difficult because orientation was more challenging in the virtual world. MacroMicro Kinematics was integrated into the software of the virtual environment to aid in the grasping and connecting together of objects which is often difficult to accomplish with present physics engines. Even with Macro-Micro Kinematics manipulation software used to simplify micro movements, the handling of virtual objects was not as precise and simple as it is in the real world [3]. Therefore it was decided to attempt to model user reaching and manipulative movements since they seemed to show different characteristics. In addition some rehabilitative virtual environments utilize faster twitch motion in game like play, so it was decided to analyze twitch movements as well [14].

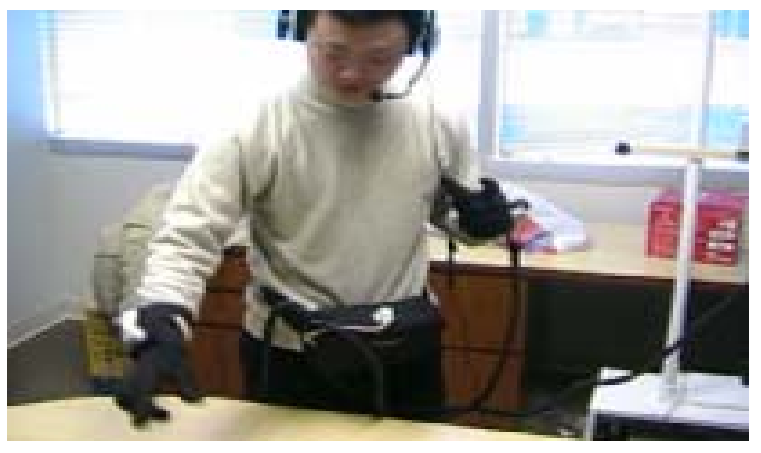

Fig 2: User Interaction in Virtual Environment

Once an adequate amount of open ended testing had been done, it was decided that more precise data needed to be collected if a rigorous model of movement was to be made. To collect the movement data five individuals were asked to perform a multitude of tests with an experimenter signaling the task to be done. The movement information was logged on the computer and processed once the experiment was completed. In addition various tasks done during open ended tested had been logged and were used as a comparison to the more scripted results.

\section{MOVEMENTS}

The first type of movement analyzed was reaching movement in which the user was to reach for a specific location on top of a table. To analyze reaching movement a user was first asked to place their hand on the center of the table in the virtual environment. A short time later a block appeared randomly on the table. The user was instructed to reach for the block and touch it when the block appeared on the table. In addition it was 
emphasized to the user that the speed at which they touched the block was not important, only that they reached and touched the block. After each test the user centered their hand on the table and then waited for the next block to appear. A slight modification to this exercise was made by instructing the tester to make sure that they touch the block. Since orientation in a virtual environment is more difficult, this required the tester to use very precise movements. To gage how different reaching lengths effected the movement the distance of the block from the center of the table was varied from being relatively short to half the length of the table.

Next manipulative movement was analyzed by having the user stack three blocks. As before the user started their hand on the center of the table in the virtual environment. Beside the user's hand three blocks were stacked on top of each other. When signaled by the instructor, the user was asked to re stack the three blocks onto a target on the center of the table where their hand had previously rested. It was important that the blocks were stacked on top of each other, but the user was instructed not to worry about how neatly and accurately the blocks were stacked.

The last type of movement analyzed was twitch movement. Even though there are many different types of twitch movements in sports and games, only one was chosen for the experiment. The user was instructed to start their hand on the center of the virtual table as before, and to catch or block a block as it fell towards the table. It was not vital as to how the user grasped or touched the block as the movement being studied was that of the twitch movement of the arm and hand. This type of experiment was chosen as it could mimic many twitch movements a user would perform when seated at a desk or table in a CVE.

Finally all of the previous three experiments were performed in the real world with the data gloves and trackers only used to measure the position information. The system was calibrated so that the moves made in the real world correspond to the same distance moved in the virtual world. The main differentiating factor between the experiments was that the user was better able to orient themselves in the real world and manipulative movements with tactile feedback in the real world were much easier to perform. As before the position information was logged and processed to see how a model of human movement in the real world might differ from that done in the virtual world.

\section{RESULTS}

To validate the results seen in open ended experimentation, multiple trial runs of the different experiments were performed and recorded. Each set of data for each trial was collected, parsed, and graphed so that trends could be found in the user's movement patterns. Velocity was used as the primary metric since it has been shown to follow regular Gaussian patterns in reaching movement in other fields of research. Although users show slight variations such as the peak speed of their movement, the shape of the user's velocity followed somewhat regular patterns and in some cases almost perfectly Gaussian as shown in Fig. 3. The data presented is a snapshot of one user's results that reflect a typical user.

When reaching movement was performed in the real world and in the virtual world the results were closer than other tests to what was expected from previous research. In both cases the speed of the user's hand followed a Gaussian speed profile fairly well. In a few circumstances the speed of the user's hand in the virtual world showed a second smaller peak as shown in the top graph of Fig. 4. The most likely cause for this is the user slightly adjusting their movement because of orientation difficulties in the virtual world. Since complete immersion in the virtual world is difficult to achieve often a user is not aware of their precise location and interaction with objects can become more difficult. There are various reasons for this orientation difficulty and lack of orientation ranging from lack of detailed graphics, lack of tactile feedback, and the delay in response time between the real world and the virtual world.

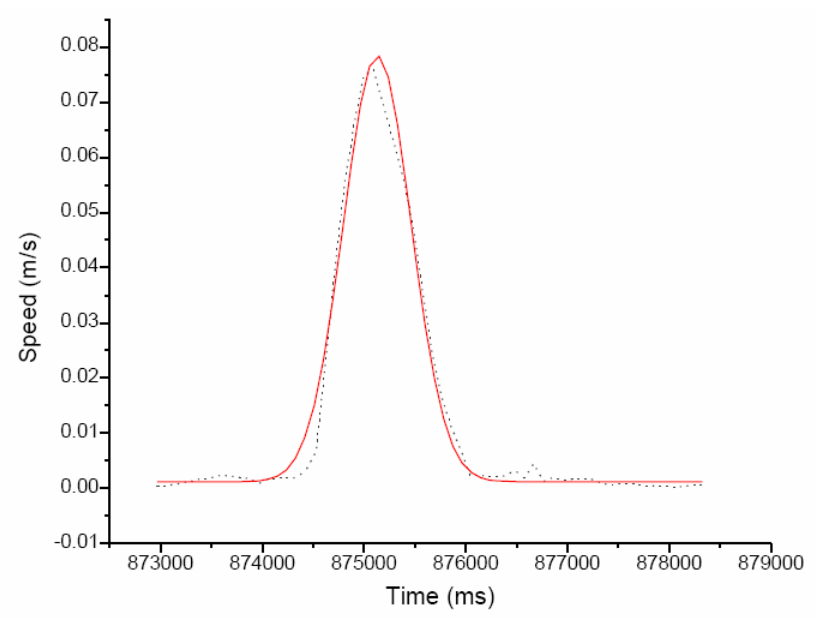

Fig 3: Gaussian Fit of Reaching Movement

Since reaching movement followed a Gaussian speed profile, it was expected that the tested twitch movement would as well. The twitch movement involved catching or blocking a falling block which would be similar to a user reaching toward a position except at a higher rate of speed. In the case of real twitch movement this was somewhat true since the movement had a roughly Gaussian shape in most of the trials. But the twitch movement exhibited less of a smooth Gaussian speed profile, and in some cases there was a large spike in the speed.
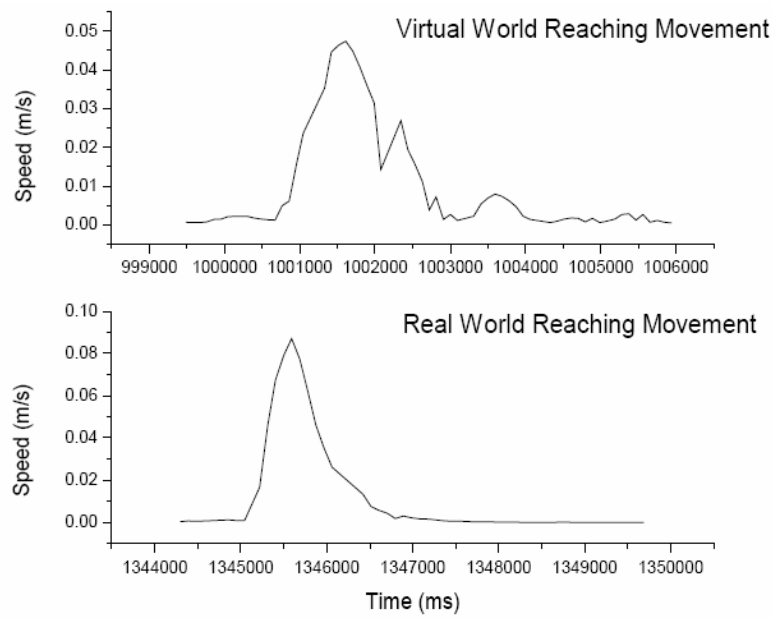

Fig 4: Reaching Movement in the Real and Virtual World

When the same experiment was tried in the virtual world, the Gaussian shape degraded even further. Clearly increased 
acceleration will lessen the Gaussian shape of the speed, but the large spikes observed in the virtual environment could point to other difficulties. Once again the delay from user action to when the action is displayed in the virtual environment cause the user to overreact and shoot their arm out wildly. As the speed of interaction in the virtual environment increase it can be deduced that the delay between the input and the software execution could cause more pronounced degradations.
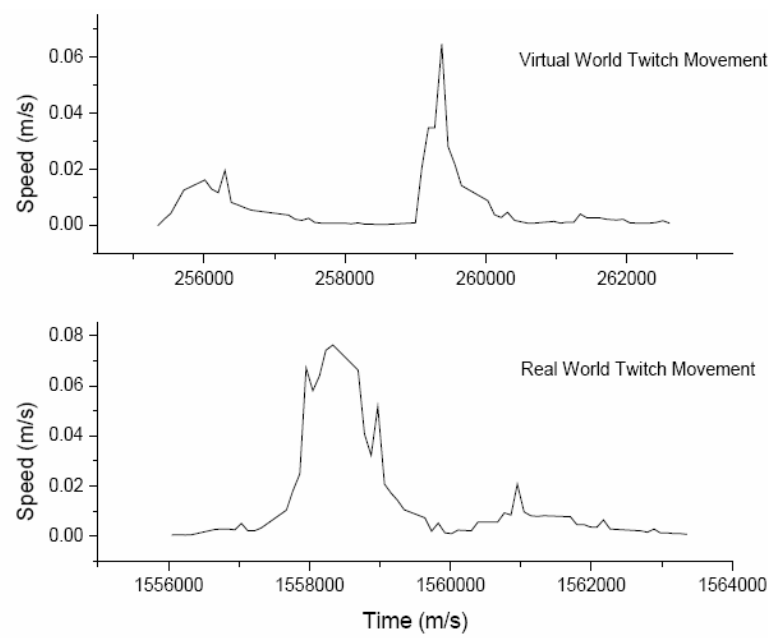

Fig 5: Twitch Movement in the Real and Virtual Environment

The last task tested was the stacking of three blocks which required great dexterity and small arm manipulations. This experiment illustrated the greatest difference between movement in the real world and that in the virtual world. For most users the tester noticed much more difficulty the users had when trying to stack the blocks in the virtual world compared to the real world. In both cases the movement could roughly be modeled as having a Gaussian speed profile, but typically with a large spike indicating a tendency for the user to change their hand speed abruptly.
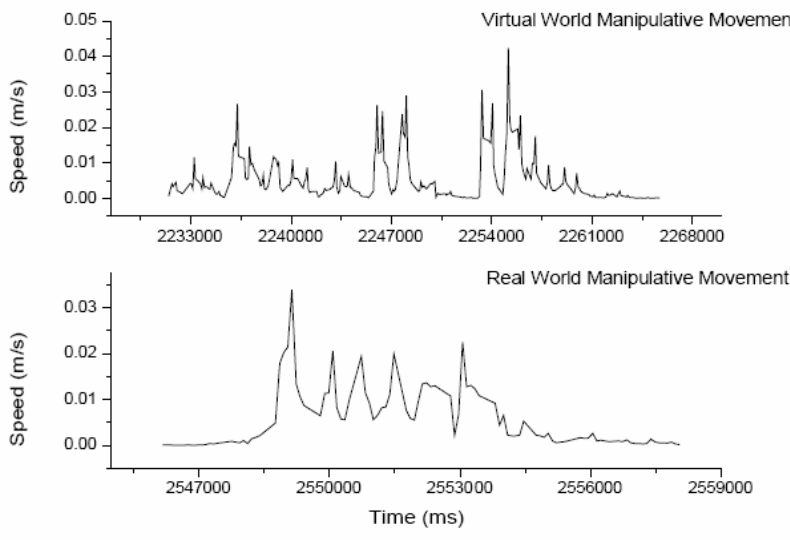

Fig 6: Manipulative Movement in the Real and Virtual World

As in the previous tasks the more difficult task of orientation in the virtual world likely contributed to the increase number of spikes. Another factor leading to the increased number of spikes was the shorter distances traveled in this task. Since the user was only making short movements the delay between their movement and it showing up the virtual environment had a greater impact on performance. The users had to be very careful not to overshoot their destination which they often did.

Another difference between the movement in the virtual world compared to the real world was an increase in the number of Gaussian like shapes. In the real world six distinct Gaussian like shapes can be seen representing the user moving their hand towards the block, grasping it, and then move the block to the center of the table. Instead of having six distinct peaks, the virtual world had roughly nine peaks showing how the user often either overshoot the goal or moved a short distance before reorienting themselves and moving again. The wait and see trick users utilized in the virtual world is illustrated with the velocity often peaking and then coming back to zero as the user tries to reorient themselves. In the real world the user tends to keep moving while performing a task and the velocity rarely return to zero. Both the frequent peaks and the more numerous valleys show how manipulative movement in a virtual environment exhibits more jerkiness than that of its real world counterpart.

\section{DISCUSSION}

By analyzing human movement in the performance of tasks a model of human movement can be developed and documented. As illustrated in the preceding section some movements such as human arm reaching better follow the expected model than other more intricate movements. Although all movements did not perfectly match the model, all movements still exhibited a roughly Gaussian speed profile. With this generic model of human movement software can be designed to estimate or predict future positions from the current position and movement data.

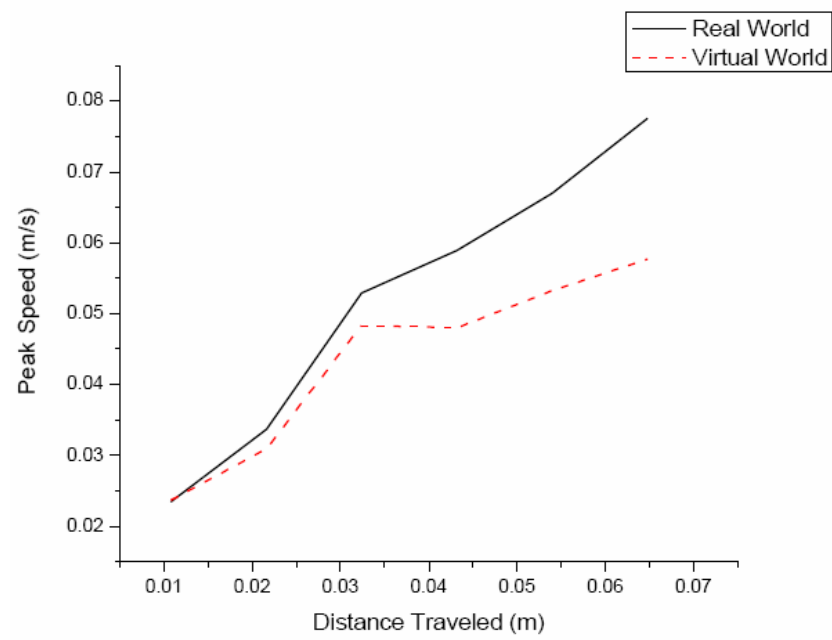

Fig 7: Peak Arm Speed Versus the Distance Traveled

The most obvious observation was that longer reaching movements tended to have higher peak speeds than shorter movements. To test the validity of this, the peak speed for different length movements was measured in three separate trials and the average peak speed was plotted versus the length of the movement. Shown in Fig. 7 the data supports the visual 
observation lending credibility to a more rigorous mathematical analysis.

If predictive techniques are to be utilized the accuracy of the prediction will be dependent on how good the model estimates human movement and how close the particular user's movements are to the model. Since the software knows an estimate of a user's intended next position, the software can move the user's avatar before new state information is received from the sensors or across the network. Moving the avatar beforehand will help to reduce any effect delay and jitter cause to the state information received. This is particularly useful in networking of CVEs because many CVEs have a network delay exceeding $100 \mathrm{~ms}$. If a networked virtual environment had to wait for each new state information packet to be received before the user's avatar moved then CVEs utilizing congested networks or long distance links would be impossible. Proper prediction allows a greater level of immersion for all users and previous users not allowed to join because of networking difficulties would have the opportunity to participate.

In addition to simply predicting the future position a good model of human movement can be used to remove noise from the system. The experiments where small manipulations had to be done highlighted the difficulty in performing intricate movements in a virtual environment. Typically the lack of complete immersion and good orientation in a virtual environment hampers a user's interaction ability. These difficulties often make the user stutter or use a stop and wait mechanism to orient themselves.

Instead of projecting a user's real movements into the virtual environment, a human movement model could be used to interpret the user's intended actions and execute those in the virtual environment. By trading the precision of the avatar for a more natural form of movement could greatly increase the user's sense of immersion in the system. The user would learn to trust their natural movements instead of applying movement in an awkward fashion to orient themselves in the virtual environment. For example if a user is reaching for an object on the table in the virtual environment often the user will stutter attempting to adjust their orientation half way through the movement. By moving the user's avatars arm by the initial trajectory the stutter would be filtered out and only the natural movement would be exhibited.

\section{REFERENCES}

[1] Aggarwal, S., Banavar, H., Khandelwal, A., Mukherjee, S.,Rangarajan, S. Accuracy in Dead-Reckoning Based Distributed Multi-Player Games. Proceedings of the ACM SIGCOMM 2004 Workshops, Portland, OR, pp. 161-165, August 2004.

[2] Beigbeder, T., Coughlan, R., Lusher, C., Plunkett, J., Agu,E., Claypool, M. The Effects of Loss and Latency on User Performace in Unreal Tournament 2003. Proceedings of the ACM SIGCOMM 2004 Workshops, Portland, OR, pp. 161165, August 2004.

[3] Bouzit, M., Junag, B., and AlRegib, G. MMK: 3D Manipulation Using Macro-Micro Kinematics, Proc. of the HCII Conference, Las Vegas, NV, July, 2005.

[4] Bowman, D., Johnson, D., Hodges, L. Testbed Evaluation ofVirtual Environment Interaction Techniques. Presence (Feb. 2001), 75-95.
[5] Chen, X., Xu, G., Chen, C., Chen, C. Study on Effects of Network Characteristics on Cooperative Performance in a Desktop CVE System. 8th International Conference on Computer Supported Cooperative Work in Design, Xiamen, China, pp. 121-126, May 2004.

[6] Flash, T., Hogan, N. The Coordination of Arm Movements: An Experimentally Confirmed Mathematical Model. The Journal of Neuroscience, USA, pp. 1688-1703, July 1985.

[7] Frees, S., Kessler, G. Precise and Rapid Interaction Through Scaled Manipulation in Immersive Virtual Environments. IEEE Virtual Reality 2005, Bonn, Germany, pp 99-106, March 2005.

[8] Garrett, A., Aguilar, M., Barniv, Y. A Recurrent Neural Network Approach to Virtual Environment Latency Reduction. Proceedings of the International Joint Conference on Neural Networks, Honolulu, HI, pp. 22882292, May 2002.

[9] Gunn, C., Hutchins, M., Adcock, M. Combating Latency in Haptic Collaborative Virtual Environments. Presence (June 2005), 313-328.

[10] Gutwin, C., Benford, S., Dyck, J., Fraser, M., Vaghi, I, Greenhalgh, C. Revealing Delay in Collaborative Environments. Conference on Human Factors in Computing Systems, Vienna, Austria, pp. 503-510, April 2004.

[11] Hanawa, D., Yonekura, T. Relationship Between Network Latency and Information Quality in a Synchronized Distributed Virtual Environment. IEEE Virtual Reality 2004, Chicago, IL, pp. 227-228, March 2004.

[12] Kim, H., Kim, S. An Improvement of Dead Reckoning Algorithm Using Kalman Filter for Minimizing Network Traffic of 3D On-line Games. 6th Pacific Rim Conference on Multimedia - Advances in Mulitmedia Information Processing, pp 676-687, Nov. 2005.

[13] Park, K., Kenyon, R. Effects of Network Characteristics on Human Performance in a Collaborative Virtual Environment. Proceedings of the 1999 IEEE Virtual Reality, Houston, TX, March 1999.

[14] Piron, L., Tonin, P., Piccione, F., Iaia, V., Trivello, E., Dam, M. Virtual Environment Training Therapy for Arm Motor Rehabilitation. Presence (Dec. 2005), 732-740.

[15] Quax, P., Monsieurs, P., Lamotte, W., Vleeschauwer, D., Degrande, N. Objective and Subjective Evaluation of the Influence of Small Amounts of Delay and Jitter on a Recent First Person Shooter Game. Proceedings of the ACM SIGCOMM 2004 Workshops, Portland, OR, pp. 152-156, August 2004.

[16] Ruddle, R., The Effect of Environment Characteristics and User Interaction on Levels of Virtual Environment Sickness. IEEE Virtual Reality 2004, Chicago, IL, pp. 227-228, March 2004.

[17] Smed, J., Kaukoranta, T., Hakonen, H. Aspects of Networking in Multiplayer Computer Games. Electronic Library, Emerald, pp. 87-97, 2002. 